\title{
Perspectivas e principais alterações no DSM-5
}

\section{Prospects and key changes in DSM-5}

\author{
Janaína Bandeira ${ }^{1}$. Eugênio de Moura Campos ${ }^{2}$. \\ 1 Médica, residente de Psiquiatria do Hospital Walter Cantídio (HUWC), Universidade Federal do Ceará (UFC), Fortaleza, Ceará, \\ Brasil. 2 Doutor em Farmacologia, Professor Adjunto de Psiquiatria da Universidade Federal do Ceará (UFC), Fortaleza, Ceará, \\ Brasil.
}

\section{RESUMO}

Introdução: com o avanço das pesquisas em neurobiologia e a necessidade de optimização dos critérios diagnósticos, a $5^{\text {a }}$ edição do Manual Diagnóstico e Estatístico de Transtornos Mentais (DSM-5) realizou importantes mudanças no modo de classificar, catalogar e diagnosticar as doenças mentais. Material e métodos: empreendemos pesquisa de artigos na Pubmed com análises sob diferentes perspectivas com o propósito de obter uma ampla visão das limitações e conquistas com sua publicação. Desenvolvimento: a nova edição do manual efetuou inúmeras alterações em relação à anterior, como a mudança do sistema de classificação multiaxial para dimensional, a fim de melhor abordar os sintomas como um continuum de intensidade, e a elaboração de critérios com maior especificidade para reduzir os diagnósticos "sem outra especificação". Por fim, citamos o projeto Research Domain Criteria Project (RDoC), ressaltando o contexto e os motivos de sua criação em torno do DSM-5. Conclusão: a atualização conseguiu apenas parcialmente seu objetivo de adotar amplamente o sistema dimensional e não obteve sucesso em incluir critérios genéticos ou neurobiológicos. Contudo, sua possibilidade de contínua revisão possibilita que seja renovada de acordo com as descobertas relevantes.

Palavras-chave: Manual diagnóstico e estatístico de transtornos mentais. Transtornos mentais. Psicopatologia.

\section{ABSTRACT}

Introduction: with the advancement of research in neurobiology and the need for optimization of diagnostic criteria, the 5th edition of the Diagnostic and Statistical Manual of Mental Disorders (DSM-5) made important changes in order to classify, catalog and diagnosing mental illness. Methods: undertake research articles in Pubmed with analysis from different perspectives in order to get a broad view of the limitations and achievements with its publication. Development: the new edition of the manual made several changes from the previous one, like changing the multiaxial classification system for dimensional, in order to better address the symptoms as a continuum of intensity, and the development of more specific criteria to reduce the diagnostic "not otherwise specified". Finally, we mention the RDoC project, highlighting the context and the reasons for its creation around the DSM-5. Conclusion: the update only partially achieved its goal to widely adopt the dimensional system and was not successful in including genetic or neurobiological criteria. However, their ability to continuous review enables it to be renewed in accordance with the relevant findings.

Keywords: Diagnostic and statistical manual of mental disorders. Mental disorders. Psychopathology.

Autor correspondente: Janaína Bandeira, Rua Dinamarca 387, Parangaba, Fortaleza, Ceará. CEP: 60710-570. Telefone: +55 85 99936-9695. E-mail: janabandeira@outlook.com

Conflito de interesses: Não há qualquer conflito de interesses por parte de qualquer um dos autores.

Recebido em: 18 Abr 2016; Revisado em: 09 Ago 2016; Aceito em: 17 Ago 2016. 


\section{INTRODUÇÃO}

$\mathrm{Na}$ Psiquiatria, o diagnóstico é tão importante quanto em qualquer outra área da Medicina. Através dele, permite-se predizer curso, prognóstico e planejar linhas de tratamento a curto e médio prazos, além de facilitar a comunicação entre psiquiatras e médicos de outras áreas. ${ }^{1}$

O uso do Manual Diagnóstico e Estatístico de Transtornos Mentais(DSM) é mais frequente na prática clínica nos EUAe em pesquisa. A maioria dos outros países utilizam a Classificação Internacional de Doenças e Problemas Relacionados à Saúde (CID), reservando aquele apenas para pesquisa e publicação de artigos por ser mais comum ou obrigatório em algumas revistas científicas, principalmente norte-americanas.

Publicado em maio de 2013, a 5 a edição do DSM trouxe importantes alterações em relação à sua anterior, como mudança do sistema de classificação das doenças (de multiaxial para categorial-dimensional) e possibilidade de ser continuamente renovado. Portanto, esse seria o motivo para, a partir de então, ulitizar-se algarismo arábico em vez de romano, pois tem a intenção de ser um documento com atualizações regulares. Possivelmente, teremos DSM's 5.1 e 5.2 e suas renovações. ${ }^{2}$

Este estudo é uma revisão literária dos artigos mais impactantes que foram publicados após o lançamento do DSM-5 e que lhe teceram críticas ou descreveram suas principais mudanças em relação à edição anterior.

\section{MATERIAL E MÉTODOS}

Como ferramenta de busca de periódicos, foi utilizado o sítio Pubmed através de termos como "changes from DSM-IVTR to DSM-5", "overview of DSM-5 changes", "history of DSM classification", "DSM-5 criteria" e "DSM-5 changes implications". Os artigos com informações pertinentes a esse estudo foram aproveitados para o desenvolvimento do mesmo e citados na seção de Referências.

O trabalho clássico de Robins e Guze, de 1972, sobre critérios diagnósticos em psiquiatria, também foi citado devido à sua importância.

Ainda, buscamos em livros de psiquiatria clínica sobre a história dos sistemas de classificação, seu contexto e implicações na prática.

\section{DESENVOLVIMENTO}

Desde a Antiguidade, tentou-se criar sistemas de classificação de doenças mentais de forma que os profissionais de saúde pudessem se comunicar entre si, permitir identificar os casos que mereciam tratamento, direcionar a terapêutica e avaliar a gravidade. No início do século XVIII, psiquiatras franceses e alemães iniciaram uma busca pela etiologia dos transtornos mentais na tentativa de encontrar substratos orgânicos para essas patologias. Como as pesquisas foram infrutíferas nesse fim, no final do século XIX iniciou-se a adoção de critérios clínicos para o diagnóstico. Aliado à expansão da neurociência, iniciou-se nos
Estados Unidos um movimento para aperfeiçoar o diagnóstico psiquiátrico. Dessa forma, surgiram sistemas baseados em taxonomia, ou seja, sistemas de classificação científica baseados no processo de agrupamento das entidades com característicaschave que compartilhem de conceitos em comum. ${ }^{3}$

Kraepelin, por exemplo, propôs que os quadros psicóticos poderiam ser diferenciados pela sua psicopatologia e evolução clínica. As classificações modernas, como o DSM e a CID, são consideradas "neokraepelianas" por seguirem as bases desse modelo. ${ }^{3}$

Ainda, Kraepelin organizava as doenças mentais utilizando o sistema de classificação vertical, no qual elas eram hierarquizadas. Se houvesse evidências de causas orgânicas, isso suplantaria outras considerações, independente do conjunto de sintomas psiquiátricos que o paciente pudesse apresentar. ${ }^{4}$ Tal modelo foi responsável por moldar os DSM's I e II.

Robins e Guze, em seu trabalho de 1972, sustentavam que a melhor maneira de se diagnosticar em Psiquiatria seria pelo estudo da história natural da doença. Para tanto, criaram 5 critérios diagnósticos: descrição clínica; testes laboratoriais; delimitação com outros diagnósticos (através de critérios de exclusão); estudos de acompanhamento; e histórico familiar. ${ }^{1}$ Criticaram a maneira categorial e limitada dos manuais diagnósticos da época, culminando em mudanças significativas no DSM subsequente (no caso, a terceira edição), como a adoção do sistema multiaxial a fim de contemplar os aspectos sociais, cognitivos e funcionais dos pacientes. ${ }^{4}$

Há 5 tipos de classificação de doenças, utilizadas nas diversas áreas da Medicina. O DSM-IV utilizou o sistema de classificação multiaxial com a finalidade de proporcionar múltiplas visões do funcionamento do paciente e de sua doença, tendo sido considerado um avanço no diagnóstico psiquiátrico na época. Cada eixo era independente, podendo-se abordar características como síndrome psiquiátrica, personalidade, doenças físicas, aspectos psicossociais e funcionais. ${ }^{5}$

Já o DSM-5 propõe a classificação dimensional na descrição de suas patologias. Para justificar o abandono do sistema de classificação multiaxial para o dimensional, afirma que: evidências científicas atualmente colocam vários transtornos, ou mesmo a sua maioria, em um espectro com transtornos intimamente relacionados que apresentam sintomas compartilhados, fatores de risco ambientais, genéticos e possivelmente substratos neuronais compartilhados [...]. Em suma, reconhecemos que os limites entre os transtornos são mais permeáveis do que se concebia anteriormente. ${ }^{6}$

Assim, afirma que os indivíduos podem apresentar poucos sintomas e estarem no limite da normalidade, ou se encontrarem entre dois transtornos, como o psicótico e o afetivo, além de ter se tornado mais valorizada com o avanço do conhecimento acerca dos aspectos dimensionais de mensuração das doenças mentais. As avaliações desse modelo estendem-se a outras características além dos sintomas, como comportamentos desadaptativos, traços temperamentais e alterações da 
funcionalidade. Os sintomas são vistos como um continuum de intensidade, reduzindo a imposição de pontos de corte para um limiar patológico. ${ }^{6}$

O sistema multiaxial foi abandonado em razão de ser pouco utilizado na prática clínica e devido ao avanço de ferramentas que avaliam longitudinalmente os sintomas e o aspecto clínico do paciente ${ }^{6}$ tornando o sistema dimensional supostamente mais adequado atualmente.

No sistema categorial, utilizado tradicionalmente nas demais áreas da Medicina, há adoção de limites entre saúde e doença, normalidade e subnormalidade. Pela descrição concreta das doenças, torna-se mais fácil realizar o diagnóstico, tornando-o mais aceito entre os profissionais de saúde devido à facilidade do seu uso (porém, bastante limitada ao ignorar quadros de subnormalidade e ser pouco útil na avaliação da gravidade e prognóstico) ${ }^{5}$

No DSM-5, a organização dos capítulos reflete melhor uma abordagem cronológica do ciclo vital, figurando o capítulo de transtornos mentais mais comuns na infância/adolescência inicialmente, e os transtornos neurocognitivos, mais vistos na idade adulta, no fim do manual. ${ }^{6}$ Fatores de risco genético e fisiológicos e indicadores prognósticos encontram-se em destaque no texto. Especificadores para determinadas patologias foram acrescentados, de forma a evitar que múltiplos diagnósticos fossem realizados, como o especificador "com sintomas ansiosos" para o transtorno depressivo maior.

Para os de personalidade, chegaram à conclusão de que são melhor representados como um continuum, pois o normal e o anormal não são muito distintos. ${ }^{8}$ Assim, o eixo II do antigo DSM-IV foi dissolvido entre as seções I e III da atual edição por não haver base biológica, psicométrica ou psicológica que justificasse sua separação dos demais. ${ }^{9}$

Ainda, reduziu o número de casos "sem outra especificação" ao criar critérios diagnósticos com maior especificidade. As edições anteriores concentravam-se em excluir diagnósticos falso-positivos, tornando as categorias extremamente restritas e a necessidade difundida do diagnóstico "sem outra especificação". Seguindo contra o fluxo das edições anteriores, as definições para as diferentes doenças foram reduzidas com o intuito de melhor se adaptar à classificação dimensional em vez da multiaxial. ${ }^{6}$

Além disso, o DSM-5 tem a proposta de alinhar-se com a ainda inédita CID-11, que tem data prevista de lançamento em 2016. Ambos foram progressivamente desenvolvidos em paralelo, com muitos pesquisadores trabalhando em ambos os projetos. ${ }^{3}$ A maioria das discrepâncias mais proeminentes entre as classificações não reflete diferenças científicas reais, e sim subprodutos históricos de processos de comitês diferentes. É importante salientar que o DSM é usado amplamente como ferramenta diagnóstica nos Estados Unidos, apesar de, por motivos de lei e seguridade, adotarem ainda a CID-10 para essas funções. Infelizmente, a semelhança entre os manuais é pobre, motivo pelo qual lutou-se para que suas disparidades sejam reduzidas a partir do lançamento da nova edição da CID.
Para que o DSM-5 possuísse um tamanho manejável e fosse, ao mesmo tempo, completo e com maior relevância na utilidade clínica, foram incluídos escalas e instrumentos de mensuração mais importantes na versão física, sendo outras disponíveis on-line (www.psychiatry.org/dsm5).

Quanto às críticas à atual edição, vários aspectos precisam ser explanados. Por exemplo, a ampla adoção do sistema dimensional em todo o manual não foi possível por razões pragmáticas, mas também por preocupações com possíveis incompatibilidades com os guidelines já publicados até então e as licenças do uso de determinadas substâncias. Dessa forma, o sistema categorial foi aplicado nesses casos. A abordagem multidimensional do DSM-5 é bastante modesta e pouco sofisticada, apesar da disponibilidade de escalas psicométricas. ${ }^{3}$

A maioria dos psiquiatras julgaram a adoção da dimensionalidade prematura e pouco desenvolvida até então, o que pode comprometer sua aceitação no meio científico e levá-la ao fracasso. ${ }^{10}$

O Instituto Nacional de Saúde Mental dos Estados Unidos (NIMH), um dos principais financiadores nessa área de pesquisa do país, abandonou, oficialmente, o DSM-5 duas semanas antes de ser publicado. O presidente, Thomas Insel, publicou no site do instituto em 29 de abril de 2013:11 Enquanto o DSM está sendo falado como uma 'bíblia' na área, não passa, na melhor das hipóteses, de um dicionário, criando denominações e descrevendo cada uma delas. O reconhecimento das edições tem se baseado na confiança diagnóstica - cada uma se assegurava que os clínicos usavam os mesmos termos nas mesmas situações. A fragilidade nesta edição é na falta de confiabilidade. Diferente de doenças como doença cardíaca isquêmica, linfoma ou AIDS, os diagnósticos do DSM são baseados em um consenso de grupos de sintomas, não em mensurações laboratoriais ou de imagem objetivas. No resto da Medicina, isso seria equivalente a criar sistemas diagnósticos baseados na dor torácica ou na intensidade da febre. De fato, essa tradição, comum nas demais áreas da Medicina, foi abandonada nos últimos 50 anos por concluírem que apenas os sintomas raramente indicam a melhor opção terapêutica para aquele transtorno. Pacientes com transtornos mentais merecem mais. O NIMH lançou o Research Domain Criteria Project (RDoC) para transformar o diagnóstico ao incorporar aspectos genéticos, exames de imagem, ciência cognitiva e outras fontes de informação para um novo sistema de classificação. [Tradução da autora]

Dr. David Kupfer, presidente do grupo responsável pela criação do DSM-5, admitiu que atualmente não temos conhecimento neurocientífico e biológico suficientes a ponto de serem incorporados como critérios diagnósticos. ${ }^{12}$ Dessa forma, uma das propostas do RDoC, ainda a ser lançado, é ampliar o aspecto dimensional, subutilizado até então nos manuais já lançados, através da psicopatologia dos transtornos mentais e de suas vias neurobiológicas.

Ainda, em relação aos manuais em vigor, ao descreverem as doenças através apenas de sinais e sintomas, os mecanismos disfuncionais não são adequadamente valorizados, e, portanto, não há estudos para novos tratamentos direcionados a eles. À vista disso, 
o NIMH lança o projeto RDoC a fim de criar estruturas e maneiras de estudar a fisiopatologia dos transtornos mentais, focando nos aspectos genéticos e neurocientíficos, ${ }^{13}$ uma vez que um dos grandes defeitos dos manuais DSM/CID é falta de validade. ${ }^{14}$

Ambos se tornaram padrões para a prática clínica, para a publicação internacional de artigos científicos, além de guia para processos administrativos, previdenciários e legais. Consequentemente, mudanças substanciais nos seus critérios diagnósticos teriam grandes repercussões em agências públicas e privadas, acarretando uma enorme resistência em alterações estruturais, ${ }^{12}$ todavia necessárias para maiores avanços na abordagem e tratamento dos transtornos e adequação às atuais necessidades nos campos da pesquisa e da saúde pública.

No momento, o RDoC não tem o objetivo de ser um novo manual classificatório de transtornos mentais, mas sim uma maneira de auxiliar pesquisadores para efetuarem mudanças nos já existentes. ${ }^{12}$ Essa proposta vai de acordo com o DSM5 ser um "documento vivo", em processo constante de modificações, ao contrário de suas edições anteriores. ${ }^{14}$

\section{CONSIDERAÇÕES FINAIS}

Apesar de todos os esforços, o consorte do DSM-5 não conseguiu basear os transtornos mentais em evidências neurobiológicas, permanecendo o diagnóstico fundado no

\section{REFERÊNCIAS}

1. Feighner JP, Robins E, Guze SB, Woodruff RA Jr, Winokur G, Munoz R. Diagnostic criteria for use in psychiatric research. Arch Gen Psychiatry. 1972;26(1):57-63.

2. Kupfer, DJ; Regier, DA. Neuroscience, clinical evidence, and the future of psychiatric classification in DSM-5. Am J Psychiatry. 2011;168(7):672-4.

3. Blashfield RK, Keeley JW, Flanagan EH, Miles SR. The cycle of classification: DSM-I through DSM-5. Annu Rev Clin Psychol. 2014;10:25-51.

4. Regier DA, Narrow WE, Kuhl EA, Kupfer DJ. The conceptual development of DSM-V. Am J Psychiatry. 2009;166(6):645-50.

5. Wang YP. Sistema de Classificação - Diagnóstico em Psiquiatria. In: Euripedes CM, Wagner FG, editores. Clínica Psiquiátrica. Barueri: Manole; 2011. p. 282-94.

6. American Psychiatric Association. DSM 5 - Manual Diagnóstico e Estatístico de Transtornos Mentais. 5. ed. Porto Alegre: Artmed; 2014. p. 992.

7. Möller HJ, Bandelow B, Bauer M, Hampel H, Herpertz SC, Soyka M. DSM-5 reviewed from different angles: goal attainment, rationality, use of evidence, consequences--part 1: general aspects and paradigmatic discussion of depressive disorders. Eur Arch Psychiatry Clin Neurosci. 2015;265(1):5-18.

8. Möller H-J, Bandelow B, Bauer M, Hampel H, Herpertz SC, Soyka quadro clínico e curso da doença, com raras exceções. Mesmo os marcadores mais estudados, como os relacionados à proteína beta-amiloide na doença de Alzheimer, não têm base suficiente para serem adicionados aos critérios necessários ou suficientes.

Contudo, é possível, em alguns diagnósticos, realizar avaliações quanto à severidade e ao impacto funcional provocado pela doença através de questionários dimensionais.

O fato de muitas expectativas para a atual edição do DSM não terem sido satisfeitas demonstra que uma classificação baseada principalmente em neurobiologia ainda é impossível pelas limitações do conhecimento científico atual, mesmo após grandes esforços do comitê do DSM-5 para esse fim. Isso evidencia que as descobertas nesse campo ainda se encontram em ritmo lento, e muito ainda deve ser estudado e validado para que possa ser utilizado nos manuais diagnósticos.

De maneira geral, apenas alguns passos foram feitos em direção à mudança da classificação axial para dimensional, de forma que o DSM-IV foi bastante preservado.

Atualmente, não temos outra opção a não ser permanecer com as abordagens tradicionais orientadas à sintomatologia das doenças, pelo menos para uso clínico, mas tendo em mente que possuem explanações limitadas e que podem não representar a melhor explicação científica para aquele transtorno.

M, at al. DSM-5 reviewed from different angles: goal attainment, rationality, use of evidence, consequences - part 2: bipolar disorders, schizophrenia spectrum disorders, anxiety disorders, obsessivecompulsive disorders, trauma- and stressor-related disorders, personality disorders, substance-related and addictive disorders, neurocognitive disorders. Eur Arch Psychiatry Clin Neurosci. 2015;265(2):87-106.

9. Trestman RL. DSM-5 and Personality Disorders: Where Did Axis II Go? J Am Acad Psychiatry Law. 2014;42(2):141-5.

10. Adam, D. On the spectrum. Nature. 2013;496(3):6-8.

11. Insel T. Director's Blog: Transforming Diagnosis [Internet]. Rockville, MD: National Institutes of Health; 2013 [acesso em: 08 jan 2016]. Disponível em: http://www.nimh.nih.gov/about/ director/2013/transforming-diagnosis.shtml

12. Cuthbert BN. The RDoC framework: facilitating transition from ICD/DSM to dimensional approaches that integrate neuroscience and psychopathology. World Psychiatry. 2014;13(1):28-35.

13. Insel T, Cuthbert B, Garvey M, Heinssen R, Pine DS, Quinn K, et al. Research domain criteria (RDoC): toward a new classification framework for research on mental disorders. Am J Psychiatry. 2010;167(7):748-51.

14. Matcheri S K, Dost O. The journey from RDC/DSM diagnoses toward RDoC Dimensions. World Psychiatry. 2014;13(1):44-6.

Como citar:

Bandeira J, Campos EM. Perspectivas e principais alterações no DSM-5. Rev Med UFC. 2017 jan-abr;57(1):15-18. 